\title{
Usinage virtuel intégrable à une chaîne de conception robuste : les besoins de l'industrie des turbo-réacteurs
}

\author{
Christian le Calvez ${ }^{a}$, Véronique Zerrouki, Jean-François Chabot et Jö̈l Vigneau \\ Snecma Moteurs, Centre de Compétence Industriel en Usinage, RN7, BP 81, 91003 Évry-Corbeil, France
}

Reçu le 25 février 2005, accepté le 21 mars 2005

\begin{abstract}
Résumé - La maîtrise des délais de conception et la robustesse des gammes de fabrication des turboréacteurs sont aujourd'hui incontournables. Les modélisations des procédés de fonderie et de forge sont couramment mises en oeuvre lors des développements afin d'optimiser la définition pièce aux procédés cibles, mais les insuffisances de modèles d'usinage actuels se font cruellement sentir. Une démarche d'analyse globale de faisabilité d'une pièce sensible a été mise en œuvre montrant l'intérêt potentiel et les attendus de l'usinage virtuel.
\end{abstract}

Mots clés : Usinage / simulation / fabrication virtuelle

Abstract - Simulation of machining for robust design: needs of turbo jets engines industry. Reduction of design cycles and increase of the reliability of manufacturing of turbo jet engines parts lead to work simultaneously on design and manufacturing using virtual manufacturing applications. The lake of reliable simulation of machining avoids to complete virtual manufacturing while virtual forging or casting are often used. An industrial case shows the interest of developing virtual machining.

Key words: Machining / simulation / virtual manufacturing

\section{Introduction}

Les contraintes croissantes imposées au turbo-réacteur en terme de performances (poussée, rendements accrus, rejets polluants limités, bruits maîtrisés, etc.) conduisent à concevoir des solutions technologiques de plus en plus pointues. Par ailleurs, la pression économique (coûts, délais) amène à une nécessaire réduction des temps de développement. La sécurité des vols impose une maîtrise complète des turbo-réacteurs. Les méthodes de conception et d'industrialisation doivent évoluer pour permettre de relever ce triple défit de la sécurité, de la performance et de la maîtrise des coûts.

\section{L'usinage rompt la chaîne de fabrication virtuelle}

\subsection{Le couplage nécessaire entre conception} et industrialisation

Pour relever ce challenge, les écarts éventuels entre la pièce conçue et la pièce produite doivent être anticipés,

\footnotetext{
a Auteur correspondant : christian.lecalvez@snecma.fr
}

d'autant que la complexité des composants et les séries réduites interdisent le recours à des pièces de mise au point. La solution industrielle aujourd'hui la plus prometteuse est de réaliser simultanément la mise au point des composants et leur industrialisation. Pour cela, il faut très tôt être capable d'envisager la fabrication de pièces qui sont encore au stade du dessin, d'où le rôle des outils de fabrication virtuelle.

L'idée est de prévoir la géométrie, les propriétés mécaniques et le coût de revient des pièces en simulant les procédés de fabrication envisagés et de les rapprocher des données du cahier des charges initial (voir Fig. 1). Il est alors possible d'analyser les écarts et d'itérer sur la conception pour réduire l'écart à la cible. Par ailleurs, cette approche permet de mettre au point les gammes de fabrication en même temps que la définition de la pièce cible.

\subsection{L'usinage rompt la chaîne de fabrication virtuelle}

Les modèles de forgeage et de fonderie sont actuellement assez matures [1-3] pour être utilisés sur les plateaux de développement. Ils permettent de simuler non seulement les opérations de fabrication, mais aussi de 


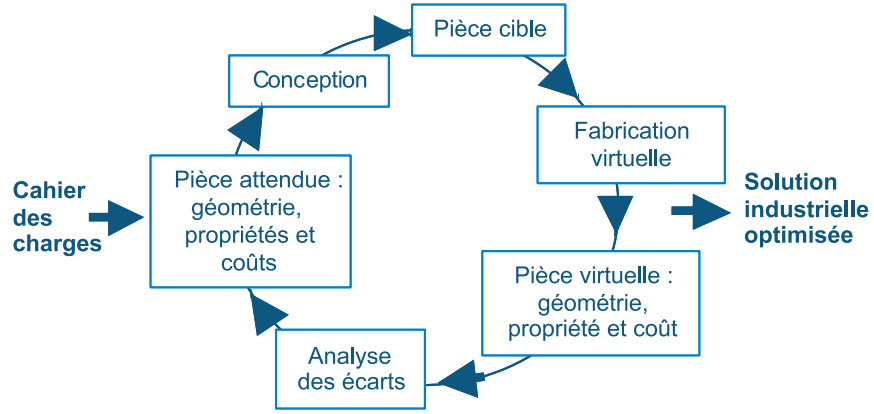

Fig. 1. Boucle de conception-industrialisation.

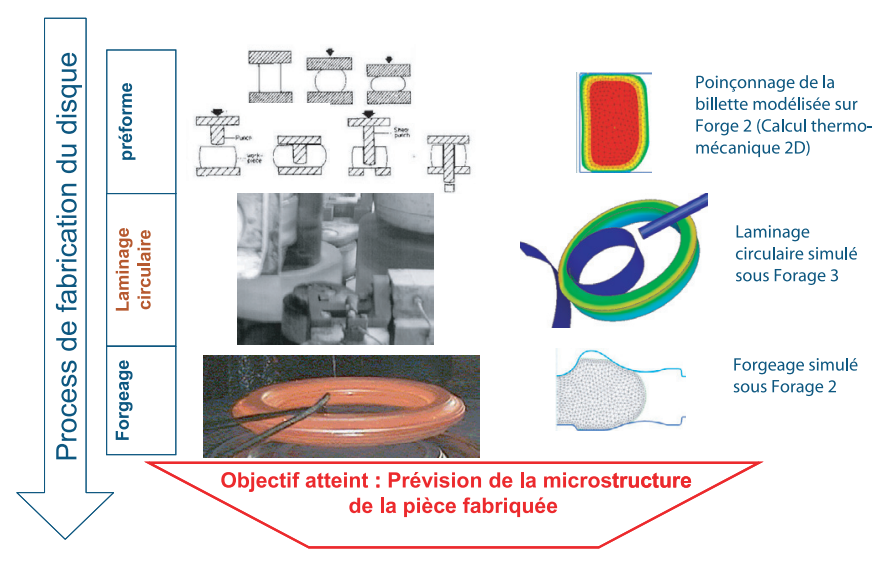

Fig. 2. Chaîne de simulation utilisée en forgeage.

prévoir les microstructures et les propriétés mécaniques pour les mettre en regard des sollicitations imposées à la pièce (voir Fig. 2). Les limites des simulations de l'usinage disponibles sur le marché ne permettent pas de rendre compte des opérations industrielles au bon niveau, et conduisent à une rupture de la chaîne de fabrication virtuelle. Ceci est d'autant plus dommageable que la grande majorité des pièces fortement sollicitées dans un turboréacteur sont obtenues par usinage d'un brut forgé.

\subsection{Les livrables attendus d'un logiciel d'usinage virtuel}

On attend d'un outil de simulation de l'usinage qu'il permette de comparer deux gammes différentes d'usinage pour choisir celle la mieux adaptée à la pièce, ou le cas échéant, de démontrer que la conception doit être revue faute de solution industrielle satisfaisante. Pour pouvoir statuer sur ces points, il faut savoir estimer :

- la qualité de la pièce (géométrie et précision accessible, l'intégrité de surface au sens état de surface, contraintes résiduelles et microstructure),

- la performance de l'opération (temps et coût de l'opération), et

- la robustesse (stabilité en fonction des paramètres de l'opération, possibilité de mettre sous maîtrise des procédés l'opération envisagée),

- un outil d'usinage virtuel permet aussi de disposer, après analyse comparative, de la gamme détaillée et des programmes machines de la solution retenue.

\section{L'usinage virtuel pluri-disciplinaire et multi-échelles}

\subsection{Liens entre les livrables et les grandeurs calculables}

Les éléments nécessaires à la comparaison des gammes d'usinage ne peuvent être estimés qu'en rassemblant des grandeurs physiques intermédiaires elles-mêmes issues de simulations des divers aspects de l'usinage (voir Fig. 3).

Pour déterminer la cote réalisée et la précision accessible, il faut disposer au premier ordre de la trajectoire de l'outil par rapport à la pièce, du positionnement et de la fixation de la pièce sur la machine (définition et variation possibles). Pour intégrer en plus les déformations de la machine il faut intégrer la rigidité de l'ensemble machine (machine, broche, bridage, pièce et outil), les efforts de coupe (et leur évolution avec l'usure de l'outil). Pour les pièces les plus précises, l'évolution de la géométrie d'arête avec l'usure d'outil peut aussi influencer la cote obtenue. Enfin, pour intégrer le retour élastique de la pièce, il faut estimer les contraintes induites par le bridage, celles existant initialement dans la pièce, ainsi que leurs évolutions du fait de l'enlèvement de matière, et celles induites en surface par l'usinage [4-6].

L'intégrité de surface de la pièce recoupe trois aspects :

- L'état de surface : sillons laissés par l'outil, des effets des vibrations, des arrachements/collage de la matière, impact de l'usure d'outil ;

- La microstructure résulte du champ de température et du champ de contraintes vus par le matériau, versus les lois de comportement du matériau sous sollicitations thermo-mécaniques;

- Les contraintes résiduelles de surface sont induites par la coupe et se déduisent des champs de déformations, vitesses de déformation et températures induites par la coupe, de l'état métallurgique en chaque point de la zone de calcul, de la loi de comportement du matériau usiné et de l'outil, et de la loi de frottement outil/matière [7-10].

Pour déterminer le temps d'opération, il faut connaître : le temps de parcours de la trajectoire (la longueur de la trajectoire dans et hors matière, les vitesses de parcours de cette trajectoire en tout point), et les temps annexes d'usinage (fréquence et temps de changement d'outils, le temps de montage, démontage et contrôle pièce).

Pour déterminer le coût de l'opération, il faut connaître : le coût machine (déduit du temps d'occupation et d'inoccupation machine et des coûts de revient de la machine), et le coût des consommables (lié aux changements d'outil et au coût de changement d'outil). 


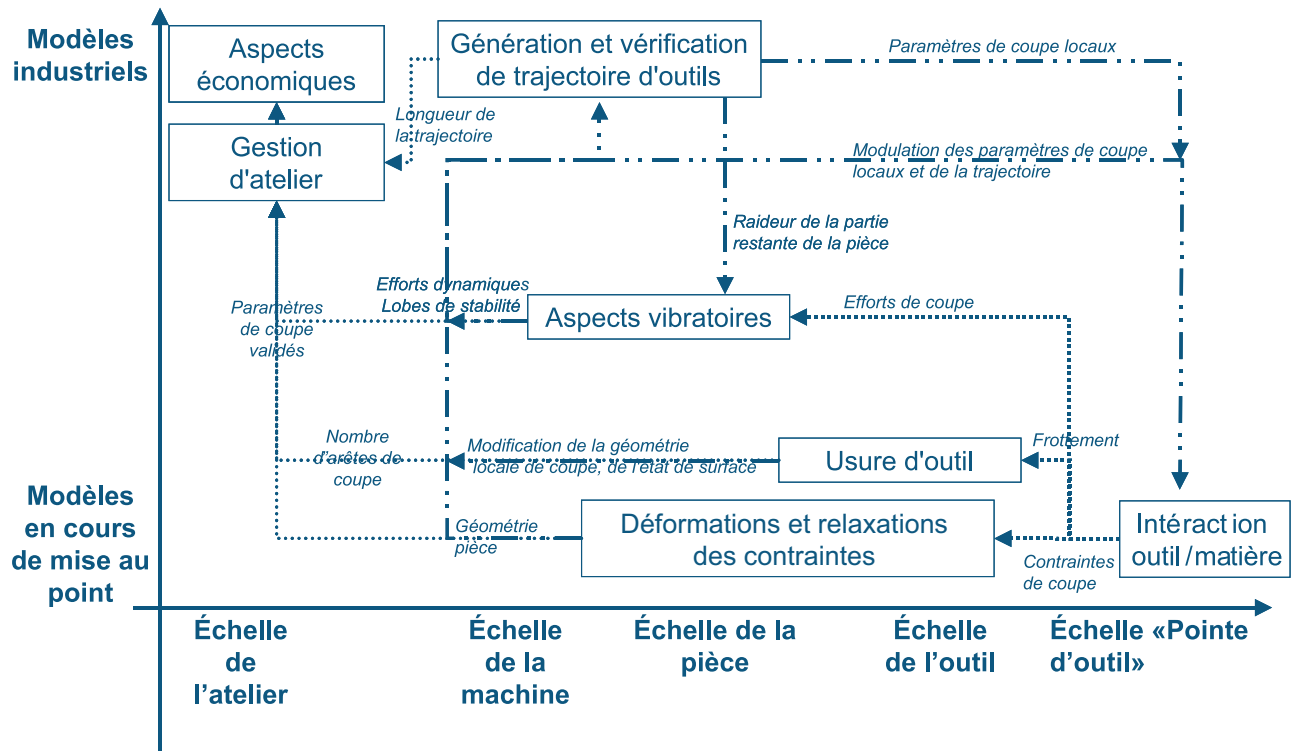

Fig. 3. Liens physiques entre les différentes échelles d'appréhension de l'usinage.

Pour estimer la stabilité de l'opération il faut connaître la précision et la fiabilité de l'ensemble des modélisations utilisées, réaliser une étude de sensibilité aux paramètres variables du procédé, et analyser les possibilités de surveillance statistique du procédé en cours d'opération.

Les grandeurs physiques à rassembler pour déterminer les livrables de l'usinage virtuel sont issues de modélisations de différents aspects de la coupe qui considèrent le phénomène à différentes échelles (voir l'axe des abscisses du graphe de la Fig. 3).

\subsection{Besoin d'une approche multi-phénomènes et multi-échelles}

Il existe des interactions fortes entre les différents aspects physiques de l'usinage. La trajectoire de l'outil sur la pièce, modulée d'éventuelles vibrations, définit les conditions de coupe locales donc l'interaction de la pointe d'outil avec la matière. L'approche locale doit permettre d'appréhender les contraintes résiduelles, mais aussi les conditions de frottement sur l'outil et les efforts de coupe. L'usure de l'outil induite par le frottement modifie ces conditions locales. L'effort de coupe est nécessaire à l'appréhension des phénomènes vibratoires, qui, lorsqu'ils n'interdisent pas l'usinage, modulent significativement la trajectoire de l'outil et donc les efforts eux-mêmes. Le calcul vibratoire nécessite aussi la connaissance de la rigidité de la pièce qui diffère selon la matière restante sur la pièce, et donc de la trajectoire d'outil retenue. Des méthodes permettant de prendre en compte ces couplages doivent être développées (voir les liens schématisés entre les différents modules sur le graphe de la Fig. 3).

\section{Besoins industriels et progrès attendus par rapport à la pratique actuelle}

\subsection{Maturité des outils existants}

Les différents modèles existants aujourd'hui pour décrire l'usinage sont de niveau de maturité très divers (voir l'axe des ordonnées du graphe de la Fig. 3). Les outils de génération ou de vérification de trajectoire sont aujourd'hui utilisés industriellement. Les modèles de recherche de stabilité vibratoire commencent à être mis en œuvre industriellement mais nécessitent encore des développements selon les applications visées [11]. La description de l'interaction pointe d'outil/pièce reste universitaire $[12,13]$, mais des approches physico-empiriques (Couple Outil Matière) permettent de border la bonne utilisation des outils dans la matière au niveau industriel [14]. La possibilité d'utiliser un modèle au niveau industriel est aussi largement liée à l'existence de modèles et de données permettant de l'alimenter ou à la possibilité de mettre en œuvre des essais simples physiques ou technologiques pour les acquérir [15-18].

\subsection{Exemple d'une démarche industrielle de réduction de risques}

Le cas industriel envisagé est celui d'une pièce d'étanchéité. C'est une pièce de révolution avec au niveau de son diamètre extérieur les surfaces d'appuis (étanchéité) et plus proche de son diamètre intérieur, les surfaces de positionnement et de fixation. Ces deux zones sont reliées entre elles par un voile mince courbe. Un précision de $\pm 0,1 \mathrm{~mm}$ sur la cote reliant la surface de positionnement (dans une zone massive), et la surface d'appuis (à l'extrémité de la zone élancée) est nécessaire pour assurer la fonction d'étanchéité. L'existence d'une partie 


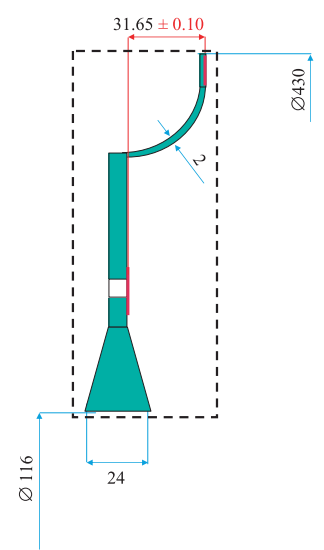

Fig. 4. Schéma de la pièce du cas type et positionnement de principe (pointillés) du brut de forge pré-usiné.

massive impose l'utilisation d'un brut de forte épaisseur (représentée par les pointillés sur la Fig. 4).

Les risques principaux identifiés pour cette pièce sont: l'incapacité à respecter la géométrie de la pièce dans les tolérances imposées, et la difficulté à usiner la pièce du fait de vibrations ou résonances des parties minces et élancées.

La levée de ces risques nécessite d'envisager d'une part toutes les sources possibles de déformations : déformations liées à la relaxation des contraintes de forge dans le brut, déformations constatées lors du relâchement des forces de bridage, et déformations liées aux contraintes d'usinage (dans la zone de faible épaisseur).

Il faut envisager d'autre part, toutes les causes racines de vibration : la rigidité de la pièce bridée sur la machine au moment de l'usinage, les fréquences propres de la pièce, les fréquences d'excitation liées à la machine, et les efforts de coupe générés par l'outil.

Il faut enfin disposer d'approches permettant d'exploiter ces grandeurs pour interpréter le niveau de risque sur la pièce.

Faute d'outils de simulation dédiés, le travail s'est déroulé sous la forme d'un tour de table d'experts cherchant à approcher les phénomènes envisagés avec des approches itératives de précision croissante.

$\mathrm{Au}$ final, le risque de déformation par relaxation des contraintes de forge a été écarté en prenant en compte les déformations constatées sur une simulation d'enlèvement de matière sur le brut de forge après trempe. La sur-épaisseur laissée en fin d'ébauche est supérieure à l'ordre de grandeur de la déformation estimée. Pour limiter les contraintes de bridage, l'expérience des pièces souples et les recommandations du groupe de travail ont conduit à un montage optimisé. Les conditions de finition (géométrie d'outil et conditions de coupe) permettent de lever le risque de fortes contraintes d'usinage. Le risque de vibration a nécessité de plus nombreuses itérations, les efforts estimés et la rigidité de la pièce finie (cas pessimiste) ne permettant pas de lever le risque. Il a fallu travailler sur une géométrie d'arête limitant les efforts de coupe, et calculer la rigidité de la pièce en fonction de l'avancement de l'usinage pour se rassurer sur ce point.
Les recommandations issues de cette analyse ont pu être formulées par le groupe de travail 5 mois après la prise en charge du problème. Ce délai n'autorise pas à travailler en boucle fermée avec la conception de la pièce, et contraint à une approche séquentielle de la conception et de l'industrialisation.

\subsection{Les attendus d'un outil d'usinage virtuel}

L'objectif industriel d'un outil de simulation est de réaliser de façon rapide et la plus automatisée possible des analyses comparables à celle présentée ci-dessus, de façon à simplifier et accélérer la démarche de levée de risques pour la rendre compatible avec une démarche itérative convergente de conception/industrialisation. Par rapport à la situation actuelle, on peut identifier deux axes de progrès : l'amélioration des modélisations des phénomènes mis en jeu par la coupe, et l'amélioration de la communication de ces modélisations entre elles.

Le besoin industriel sur ce sujet est pressant et tout progrès même partiel mérite d'être validé et industrialisé. Par ailleurs, un travail sur les formats d'échange de données et de couplage entre eux des modèles devrait permettre d'accélérer et de faciliter l'intégration entre eux des modules décrivant les différents phénomènes, intégration qui d'expérience est toujours beaucoup plus difficile à mener a posteriori qu'a priori.

\section{Conclusion}

L'utilisation d'outils de fabrication virtuelle est aujourd'hui indispensable pour optimiser simultanément la conception et l'industrialisation des pièces et répondre aux contraintes du marché des turbo-réacteurs. Il manque aujourd'hui un outil d'usinage virtuel qui permette de statuer sur la qualité attendue de l'opération, sa performance économique et sa robustesse. L'analyse des grandeurs physiques nécessaires à cette analyse montre qu'il faut prendre simultanément en compte des phénomènes physiques variés intervenant à différentes échelles et dont la description par des modèles est de maturité inégale. Dans la pratique industrielle, la levée de risque lors de l'industrialisation de pièces critiques nécessite des démarches qui restent longues et spécifiques aux cas traités. L'enjeu du développement de l'usinage virtuel est de mettre à disposition, des modules de simulation des différents aspects de la coupe, compatibles entre eux en terme d'échange de données.

\section{Références}

[1] N. Späth, V. Zerrouki, P. Poubanne, J.Y. Guedou, 718 Superalloy Forging Simulation: a way to improve process and material potentialities, International Symposium on Superalloys 718, 625, 706 and various derivatives, Pittsburg, 2001 
[2] G. Losilla, P. Montmitonnet, M. Bouzaiane, P.E. Clément, Modélisation du laminage circulaire par éléments finis : Calculs thermomécaniques et microstructuraux, Colloque Matériaux, Tours, 2002

[3] S. Ponnelle, N. Späth, T. Godon, J.Y. Guedou, Numerical prediction of microstructure and mechanical properties of 718 alloy forges engine parts: a process optimisation tool, Colloque Matériaux, Tours, 2002

[4] F. Gu, S. Kapor, R. de Vor, An enhance cutting force model for face milling with variable cutter feed motion and complex workpiece geometry, Transactions ASME J. Int. Manufacturing Sci. Eng. 119 (1997) 467-475

[5] Y.G. Liao, S.J. Hu, An integrated model of a fixtureworkpiece system for surface quality prediction, Int. J. Adv. Manufacturing Techn. 117 (2001) 810-818

[6] E. Cheung, W. Yuan, M. Hua, Physical simulation of the deflection in turning of thin disk-shaped workpiece, Int. J. Adv. Manufacturing Techn. 15 (1999) 863-868

[7] V. Kalhori, Modeling and simulation of mechanical cutting, Thèse, Lulea University of Technology, 2001

[8] C.R. Liu, M.M. Barash, Variables governing patterns of mechanical residual stress in a machined surface, J. Eng. Ind. 104 (1982) 257-264

[9] K. Okushima, K. Yoshiaki, The residual stress produced by metal cutting, Ann. CIRP 20 (1971) 13-14

[10] Z.C. Lin, W.L. Lai, H.Y. Lin, C.R. Liu, Residual stresses with different tool flank wear lengths in the ultra precision machining of Ni-P alloys, J. Mat. Processing Techn. 65 (1997) 116-126
[11] Y. Altintas, Manufacturing automation: metal cutting mechanics, machine tool vibrations and CNC design, Cambridge University Press, ISBN 0-521-65973-6, 2000

[12] K. Van Luttervelt, Final report on the activities of the CIRP Working Group Modeling of Machining Operations, 2002

[13] C.A. Van Luttervelt, T.H.C. Childs, I.S. Jawahir, S. Klocke, B.K. Venuvinod, Present Situation and Future Trends in Modelling of Machining Operations Progress Report of the CIRP Working Group Modelling of Machining Operations, Ann. CIRP 47 (1998) 587-624

[14] AFNOR Standard NFE 66-520, 1997, Working Zones of cutting Tool-Material Couple

[15] P.H. Arrazola, Simulation numérique de la coupe : importance de la loi de comportement du matériau et de la loi de friction, Thèse, École Centrale de Nantes, 2002

[16] B. Changeux, Loi de comportement pour l'usinage. Localisation de la déformation et aspects microstructuraux, Thèse, École nationale supérieure d'arts et métiers, 2001

[17] M. Meiller, J.L. Lebrun, M. Touratier, D. Ryckelyinck, Friction law for tool/workpiece contact area in dry machining, Proceedings of the international workshop on friction and flow stress in cutting and forming, 2000, pp. 101109

[18] J.Q. Xie, A.E. Bayoumi, H.M. Zbib, FEA modelling and simulation of shear localized chip formation in metal cutting, Int. J. Machine Tools and Manufacture 38 (1998) $1067-1087$ 